\title{
Severe Hepatic Injury Caused by Orlistat
}

Takeji Umemura, MD, Tetsuya Ichijo, MD, Akihiro Matsumoto, MD, Kendo Kiyosawa MD

Department of Medicine, Shinshu University School of Medicine, Matsumoto, Japan

Corresponding author: Takeji Umemura, MD, PhD. Department of Medicine, Shinshu University School of Medicine, 3-1-1 Asahi, Matsumoto, 390-8621, Japan.

Tel:+81-263-37-2634, Fax:+81-263-32-9412, E-mail: t-ume@hsp.md.shinshu-u.ac.jp

Word count 474 
To the Editor:

The reversible lipase inhibitor orlistat is a safe and effective weight-reducing agent that decreases intestinal fat absorption. The most frequently reported side effects of orlistat have been mild to moderate gastrointestinal tract adverse events including fatty/oily stools, abdominal pain, and fecal urgency. ${ }^{1}$ However, we here would like to describe a recent case of acute hepatic injury caused by orlistat use.

A 15-year-old Thai woman without remarkable medical history had taken orlistat (120 mg three times daily) for 7 days due to concerns of obesity. One week later, the patient was admitted to an emergency hospital with abdominal pain, malaise, nausea, and diarrhea, and was transferred to our hospital the following day. She had no toxic habits and denied taking any other medications or herbal remedies. She was alert, her body temperature was $37.5^{\circ} \mathrm{C}$, BMI was $20.0 \%$, white blood cell count was $2,870 / \mu$, eosinophils were $7 \%$, and platelet count was $10.3 \times 10^{4} / \mu \mathrm{l}$. The patient's serum aspartate aminotransferase and alanine aminotransferase level were surprisingly high at 8,269 IU/I and 9,976 IU/l, respectively, total bilirubin level was $1.3 \mathrm{mg} / \mathrm{dl}$, total cholesterol was 65 $\mathrm{mg} / \mathrm{dl}$, prothrombin INR was 2.98, and IgE level was $486 \mathrm{IU} / \mathrm{ml}$. Results of screening for viral hepatitis (hepatitis A, B, C, and E virus; cytomegalovirus; Epstein-Barr virus) and autoimmune markers (anti-nuclear antibodies, and anti-mitochondrial antibodies) were 
all negative. Abdominal ultrasound, computed tomography, and magnetic resonance imaging all showed normal findings. The patient was given vitamin-K intravenously for 5 days, and liver function tests normalized within 1 month.

Although possible associations between hepatotoxicity and orlistat have been reported, ${ }^{2,3}$ there have been few cases of serious adverse hepatic effects. The exact mechanism of hepatotoxicity in our patient is unknown, though the presence of low grade fever, eosinophilia, elevated IgE level, and cytopenia indicates an immunoallergic reaction. Additionally, the temporal association between institution of orlistat and the onset of hepatic abnormalities, the resolution of symptoms and improvement of abnormal liver function test after the drug was stopped, and the absence of alternative explanations strongly suggest that orlistat caused her liver injury. The Clinical Diagnostic Scale ${ }^{4}$ has also indicated a probable relationship between acute hepatotoxicity and orlistat. Taken together, our report indicates that orlistat may cause substantial acute liver injury. Clinicians should be aware of this possibility when prescribing this drug or when presented with patients taking orlistat. 


\section{References}

1. Chanoine JP, Hampl S, Jensen C, et al. Effect of orlistat on weight and body composition in obese adolescents: a randomized controlled trial. JAMA. 2005;293:2873-2883.

2. Montero JL, Muntane J, Fraga E, et al. Orlistat associated subacute hepatic failure. J Hepatol. 2001;34:173.

3. Lau G, Chan CL. Massive hepatocellular [correction of hepatocullular] necrosis: was it caused by Orlistat? Med Sci Law. 2002;42:309-312.

4. Maria VA, Victorino RM. Development and validation of a clinical scale for the diagnosis of drug-induced hepatitis. Hepatology. 1997;26:664-669. 